\title{
Strong differential subordination properties for analytic functions involving the Komatu integral operator
}

Nak Eun Cho*

\section{${ }^{*}$ Correspondence:}

necho@pknu.ac.kr

Department of Applied

Mathematics, Pukyong National

University, Busan, 608-737, Korea

\begin{abstract}
The purpose of the present paper is to investigate some strong differential subordination and superordination implications involving the Komatu integral operator which are obtained by considering suitable classes of admissible functions. The sandwich-type theorems for these operators are also considered.
\end{abstract}

MSC: $30 \mathrm{C} 80 ; 30 \mathrm{C} 45 ; 30 \mathrm{~A} 20$

Keywords: strong differential subordination; strong differential superordination; univalent function; convex function; Komatu integral operator

\section{Introduction}

Let $\mathcal{H}$ denote the class of analytic functions in the open unit disk $\mathbb{U}=\{z \in \mathbb{C}:|z|<1\}$. For a positive integer $n$ and $a \in \mathbb{C}$, let

$$
\mathcal{H}[a, n]=\left\{f \in \mathcal{H}: f(z)=a+a_{n} z^{n}+a_{n+1} z^{n+1}+\cdots\right\},
$$

and let $\mathcal{H}_{0} \equiv \mathcal{H}[0,1]$. We also denote by $\mathcal{A}$ the subclass of $\mathcal{H}[a, 1]$ with the usual normalization $f(0)=f^{\prime}(0)-1=0$.

Let $f(z)$ and $F(z)$ be members of $\mathcal{H}$. The function $f(z)$ is said to be subordinate to $F(z)$, or $F(z)$ is said to be superordinate to $f(z)$, if there exists a function $w(z)$ analytic in $\mathbb{U}$, with $w(0)=0$ and $|w(z)|<1$, and such that $f(z)=F(w(z))$. In such a case, we write $f(z) \prec F(z)$ or $f \prec F$. If the function $F$ is univalent in $\mathbb{U}$, then $f(z) \prec F(z)$ if and only if $f(0)=F(0)$ and $f(\mathbb{U}) \subset F(\mathbb{U})(c f .[1])$.

Following Komatu [2], we introduce the integral operator $\mathcal{L}_{c}^{\lambda}: \mathcal{A} \rightarrow \mathcal{A}$ defined by

$$
\mathcal{L}_{c}^{\lambda} f(z):=\frac{c^{\lambda}}{\Gamma(\lambda)} \int_{0}^{1} t^{c-2}\left(\log \frac{1}{t}\right)^{\lambda-1} f(t z) d t \quad(\operatorname{Re}\{c\}>0 ; \lambda \geq 0 ; f \in \mathcal{A})
$$

where the symbol $\Gamma$ stands for the gamma function. We also note that the operator $\mathcal{L}_{c}^{\lambda} f(z)$ defined by (1.1) can be expressed by the series expansion as follows:

$$
\mathcal{L}_{c}^{\lambda} f(z)=z+\sum_{k=2}^{\infty}\left(\frac{c}{c+k-1}\right)^{\lambda} a_{k} z^{k} .
$$

\section{Springer}

(0) 2013 Cho; licensee Springer. This is an Open Access article distributed under the terms of the Creative Commons Attribution License (http://creativecommons.org/licenses/by/2.0), which permits unrestricted use, distribution, and reproduction in any medium, provided the original work is properly cited. 
Obviously, we have, for $\lambda, \mu \geq 0$,

$$
\mathcal{L}_{c}^{\lambda}\left(\mathcal{L}_{c}^{\mu} f(z)\right)=\mathcal{L}_{c}^{\lambda+\mu} f(z)
$$

Moreover, from (1.2), it follows that

$$
z\left(\mathcal{L}_{c}^{\lambda+1} f(z)\right)^{\prime}=c \mathcal{L}_{c}^{\lambda} f(z)-(c-1) \mathcal{L}_{c}^{\lambda+1} f(z)
$$

In particular, the operator $\mathcal{L}_{2}^{\lambda}$ is closely related to the multiplier transformation studied earlier by Flett [3]. Various interesting properties of the operator $\mathcal{L}_{2}^{\lambda}$ have been studied by Jung et al. [4] and Liu [5].

To prove our results, we need the following definitions and theorems considered by Antonimo [6, 7] and Oros [8, 9].

Definition $1.1([6], c f .[7,8])$ Let $H(z, \zeta)$ be analytic in $\mathbb{U} \times \overline{\mathbb{U}}$ and let $f(z)$ be analytic and univalent in $\mathbb{U}$. Then the function $H(z, \zeta)$ is said to be strongly subordinate to $f(z)$, or $f(z)$ is said to be strongly superordinate to $H(z, \zeta)$, written as $H(z, \zeta) \prec \prec f(z)$, if for $\zeta \in \overline{\mathbb{U}}$, $H(z, \zeta)$ as the function of $z$ is subordinate to $f(z)$. We note that $H(z, \zeta) \prec \prec f(z)$ if and only if $H(0, \zeta)=f(0)$ and $H(\mathbb{U} \times \overline{\mathbb{U}}) \subset f(\mathbb{U})$.

Definition $1.2\left([8], c f\right.$. [1]) Let $\phi: \mathbb{C}^{3} \times \mathbb{U} \times \overline{\mathbb{U}} \rightarrow \mathbb{C}$ and let $h(z)$ be univalent in $\mathbb{U}$. If $p(z)$ is analytic in $\mathbb{U}$ and satisfies the (second-order) differential subordination

$$
\phi\left(p(z), z p^{\prime}(z), z p^{\prime \prime}(z) ; z, \zeta\right) \prec \prec h(z)
$$

then $p(z)$ is called a solution of the strong differential subordination. The univalent function $q(z)$ is called a dominant of the solutions of the strong differential subordination, or more simply a dominant, if $p(z) \prec q(z)$ for all $p(z)$ satisfying (1.4). A dominant $\tilde{q}(z)$ that satisfies $\tilde{q}(z) \prec q(z)$ for all dominants $q(z)$ of (1.4) is said to be the best dominant.

Recently, Oros [9] introduced the following strong differential superordinations as the dual concept of strong differential subordinations.

Definition 1.3 ([9], $c f$. [10]) Let $\varphi: \mathbb{C}^{3} \times \mathbb{U} \times \overline{\mathbb{U}} \rightarrow \mathbb{C}$ and let $h(z)$ be analytic in $\mathbb{U}$. If $p(z)$ and $\varphi\left(p(z), z p^{\prime}(z), z p^{\prime \prime}(z) ; z, \zeta\right)$ are univalent in $\mathbb{U}$ for $\zeta \in \overline{\mathbb{U}}$ and satisfy the (second-order) strong differential superordination

$$
h(z) \prec \prec \varphi\left(p(z), z p^{\prime}(z), z p^{\prime \prime}(z) ; z, \zeta\right),
$$

then $p(z)$ is called a solution of the strong differential superordination. An analytic function $q(z)$ is called a subordinant of the solutions of the strong differential superordination, or more simply a subordinant, if $q(z) \prec p(z)$ for all $p(z)$ satisfying (1.5). A univalent subordinant $\tilde{q}(z)$ that satisfies $q(z) \prec \tilde{q}(z)$ for all subordinants $q(z)$ of $(1.5)$ is said to be the best subordinant.

Denote by $\mathcal{Q}$ the class of functions $q$ that are analytic and injective on $\overline{\mathbb{U}} \backslash E(q)$, where

$$
E(q)=\left\{\xi \in \partial \mathbb{U}: \lim _{z \rightarrow \xi} q(z)=\infty\right\},
$$


and are such that $q^{\prime}(\xi) \neq 0$ for $\xi \in \partial \mathbb{U} \backslash E(q)$. Further, let the subclass of $\mathcal{Q}$ for which $q(0)=a$ be denoted by $\mathcal{Q}(a)$ and $\mathcal{Q}(0) \equiv \mathcal{Q}_{0}$.

Definition 1.4 ([8]) Let $\Omega$ be a set in $\mathbb{C}, q(z) \in \mathcal{Q}$ and $n$ be a positive integer. The class of admissible functions $\Psi_{n}[\Omega, q]$ consists of those functions $\psi: \mathbb{C}^{3} \times \mathbb{U} \times \overline{\mathbb{U}} \rightarrow \mathbb{C}$ that satisfy the admissibility condition

$$
\psi(r, s, t ; z, \zeta) \notin \Omega
$$

whenever $r=q(\xi), s=k \xi q^{\prime}(\xi)$ and

$$
\operatorname{Re}\left\{\begin{array}{l}
t \\
s
\end{array}+1\right\} \geq k \operatorname{Re}\left\{\frac{\xi q^{\prime \prime}(\xi)}{q^{\prime}(\xi)}+1\right\}
$$

for $z \in \mathbb{U}, \xi \in \partial \mathbb{U} \backslash E(q), \zeta \in \overline{\mathbb{U}}$ and $k \geq n$. We write $\Psi_{1}[\Omega, q]$ as $\Psi[\Omega, q]$.

Definition 1.5 ([9]) Let $\Omega$ be a set in $\mathbb{C}$ and $q \in \mathcal{H}[a, n]$ with $q^{\prime}(z) \neq 0$. The class of admissible functions $\Psi_{n}^{\prime}[\Omega, q]$ consists of those functions $\psi: \mathbb{C}^{3} \times \overline{\mathbb{U}} \times \overline{\mathbb{U}} \rightarrow \mathbb{C}$ that satisfy the admissibility condition

$$
\psi(r, s, t ; \xi, \zeta) \in \Omega
$$

whenever $r=q(z), s=z q^{\prime}(z) / m$ for $z \in \mathbb{U}$ and

$$
\operatorname{Re}\left\{\frac{t}{s}+1\right\} \leq \frac{1}{m} \operatorname{Re}\left\{\frac{z q^{\prime \prime}(z)}{q^{\prime}(z)}+1\right\}
$$

for $z \in \mathbb{U}, \xi \in \partial \mathbb{U}, \zeta \in \overline{\mathbb{U}}$ and $m \geq n \geq 1$. We write $\Psi_{1}^{\prime}[\Omega, q]$ as $\Psi^{\prime}[\Omega, q]$.

For the above two classes of admissible functions, Oros and Oros proved the following theorems.

Theorem 1.1 ([8]) Let $\psi \in \Psi_{n}[\Omega, q]$ with $q(0)=a$. If $p \in \mathcal{H}[a, n]$ satisfies

$$
\psi\left(p(z), z p^{\prime}(z), z^{2} p^{\prime \prime}(z), z^{2} p^{\prime \prime}(z) ; z, \zeta\right) \in \Omega
$$

then $p(z) \prec q(z)$.

Theorem 1.2 ([9]) Let $\psi \in \Psi_{n}^{\prime}[\Omega, q]$ with $q(0)=a$. If $p \in \mathcal{Q}(a)$ and

$$
\psi\left(p(z), z p^{\prime}(z), z^{2} p^{\prime \prime}(z) ; z, \zeta\right)
$$

is univalent in $\mathbb{U}$ for $\zeta \in \overline{\mathbb{U}}$, then

$$
\Omega \subset\left\{\psi\left(p(z), z p^{\prime}(z), z^{2} p^{\prime \prime}(z) ; z, \zeta\right): z \in \mathbb{U}, \zeta \in \overline{\mathbb{U}}\right\}
$$

implies $q(z) \prec p(z)$. 
In the present paper, making use of the differential subordination and superordination results of Oros and Oros [8, 9], we determine certain classes of admissible functions and obtain some subordination and superordination implications of multivalent functions associated with the Komatu integral operator $\mathcal{L}_{c}^{\lambda}$ defined by (1.1). Additionally, new differential sandwich-type theorems are obtained. We remark in passing that some interesting developments on differential subordination and superordination for various operators in connection with the Komatu integral operator were obtained by Ali et al. [11-14] and Cho et al. [15].

\section{Subordination results}

Firstly, we begin by proving the subordination theorem involving the integral operator $\mathcal{L}_{c}^{\lambda}$ defined by (1.1). For this purpose, we need the following class of admissible functions.

Definition 2.1 Let $\Omega$ be a set in $\mathbb{C}, q \in \mathcal{Q}_{0} \cap \mathcal{H}[0,1], \operatorname{Re}\{c\}>0$ and $\lambda \geq 1$. The class of admissible functions $\Phi_{\mathcal{L}}[\Omega, q]$ consists of those functions $\phi: \mathbb{C}^{3} \times \mathbb{U} \times \overline{\mathbb{U}} \rightarrow \mathbb{C}$ that satisfy the admissibility condition

$$
\phi(u, v, w ; z, \xi) \notin \Omega
$$

whenever

$$
u=q(\zeta), \quad v=\frac{k \zeta q^{\prime}(\zeta)+(c-1) q(\zeta)}{c},
$$

and

$$
\operatorname{Re}\left\{\frac{c^{2} w-(c-1)^{2} u}{c v-(c-1) u}-2(c-1)\right\} \geq k \operatorname{Re}\left\{\frac{\zeta q^{\prime \prime}(\zeta)}{q^{\prime}(\zeta)}+1\right\}
$$

for $z \in \mathbb{U}, \zeta \in \partial \mathbb{U} \backslash E(q), \xi \in \overline{\mathbb{U}}$ and $k \geq 1$.

Theorem 2.1 Let $\phi \in \Phi_{\mathcal{L}}[\Omega, q]$. If $f \in \mathcal{A}$ satisfies

$$
\left\{\phi\left(\mathcal{L}_{c}^{\lambda+1} f(z), \mathcal{L}_{c}^{\lambda} f(z), \mathcal{L}_{c}^{\lambda-1} f(z) ; z\right): z \in \mathbb{U}, \xi \in \overline{\mathbb{U}}\right\} \subset \Omega,
$$

then

$$
\mathcal{L}_{c}^{\lambda+1} f(z) \prec q(z)
$$

Proof Define the function $p(z)$ in $\mathbb{U}$ by

$$
p(z):=\mathcal{L}_{c}^{\lambda+1} f(z) .
$$

From (2.2) with the relation (1.3), we get

$$
\mathcal{L}_{c}^{\lambda} f(z)=\frac{z p^{\prime}(z)+(c-1) p(z)}{c} .
$$


Further computations show that

$$
\mathcal{L}_{c}^{\lambda-1} f(z)=\frac{z^{2} p^{\prime \prime}(z)+(2 c-1) z p^{\prime}(z)+(c-1)^{2} p(z)}{c^{2}} .
$$

Define the transformation from $\mathbb{C}^{3}$ to $\mathbb{C}$ by

$$
u=r, \quad v=\frac{s+(c-1) r}{c}, \quad \text { and } \quad w=\frac{t+(2 c-1) s+(c-1)^{2} r}{c^{2}} \text {. }
$$

Let

$$
\psi(r, s, t ; z, \xi)=\phi(u, v, w ; z, \xi)=\phi\left(r, \frac{s+(c-1) r}{c}, \frac{t+(2 c-1) s+(c-1)^{2} r}{c^{2}} ; z, \xi\right)
$$

Using equations (2.2), (2.3) and (2.4), from (2.6), we obtain

$$
\psi\left(p(z), z p^{\prime}(z), z^{2} p^{\prime \prime}(z) ; z, \xi\right)=\phi\left(\mathcal{L}_{c}^{\lambda+1} f(z), \mathcal{L}_{c}^{\lambda} f(z), \mathcal{L}_{c}^{\lambda-1} f(z) ; z, \xi\right)
$$

Hence, (2.1) becomes

$$
\psi\left(p(z), z p^{\prime}(z), z^{2} p^{\prime \prime}(z) ; z, \xi\right) \in \Omega
$$

Note that

$$
\frac{t}{s}+1=\frac{c^{2} w-(c-1)^{2} u}{c v-(c-1) u}-2(c-1)
$$

and so the admissibility condition for $\phi \in \Phi_{\mathcal{L}}[\Omega, q]$ is equivalent to the admissibility condition for $\psi \in \Psi[\Omega, q]$. Therefore, by Theorem 1.1, $p \prec q$ or

$$
\mathcal{L}_{c}^{\lambda+1} f(z) f(z) \prec q(z)
$$

which evidently completes the proof of Theorem 2.1 .

If $\Omega \neq \mathbb{C}$ is a simply connected domain, then $\Omega=h(\mathbb{U})$ for some conformal mapping $h$ of $\mathbb{U}$ onto $\Omega$. In this case, the class $\Phi_{\mathcal{L}}[h(\mathbb{U}), q]$ is written as $\Phi_{\mathcal{L}}[h, q]$. The following result is an immediate consequence of Theorem 2.1.

Theorem 2.2 Let $\phi \in \Phi_{\mathcal{L}}[h, q]$. Iff $\in \mathcal{A}$ satisfies

$$
\phi\left(\mathcal{L}_{c}^{\lambda+1} f(z), \mathcal{L}_{c}^{\lambda} f(z), \mathcal{L}_{c}^{\lambda-1} f(z) ; z, \xi\right) \prec \prec h(z),
$$

then

$$
\mathcal{L}_{c}^{\lambda+1} f(z) \prec q(z)
$$

Our next result is an extension of Theorem 2.1 to the case where the behavior of $q$ on $\partial \mathbb{U}$ is not known. 
Corollary 2.3 Let $\Omega \subset \mathbb{C}$ and $q$ be univalent in $\mathbb{U}$ with $q(0)=1$. Let $\phi \in \Phi_{\mathcal{L}}\left[\Omega, q_{\rho}\right]$ for some $\rho \in(0,1)$ where $q_{\rho}(z)=q(\rho z)$. If $\in \mathcal{A}$ satisfies

$$
\phi\left(\mathcal{L}_{c}^{\lambda+1} f(z), \mathcal{L}_{c}^{\lambda} f(z), \mathcal{L}_{c}^{\lambda-1} f(z) ; z, \xi\right) \in \Omega
$$

then

$$
\mathcal{L}_{c}^{\lambda+1} f(z) \prec q(z)
$$

Proof Theorem 2.1 yields $\mathcal{L}_{c}^{\lambda+1} f(z) \prec q_{\rho}(z)$. The result is now deduced from $q_{\rho}(z) \prec q(z)$

Theorem 2.4 Let $h$ and $q$ be univalent in $\mathbb{U}$ with $q(0)=0$ and $\operatorname{set} q_{\rho}(z)=q(\rho z)$ and $h_{\rho}(z)=$ $h(\rho z)$. Let $\phi: \mathbb{C}^{3} \times \mathbb{U} \times \overline{\mathbb{U}} \rightarrow \mathbb{C}$ satisfy one of the following conditions:

(1) $\phi \in \Phi_{\mathcal{L}}\left[h, q_{\rho}\right]$ for some $\rho \in(0,1)$, or

(2) there exists $\rho_{0} \in(0,1)$ such that $\phi \in \Phi_{\mathcal{L}}\left[h_{\rho}, q_{\rho}\right]$ for all $\rho \in\left(\rho_{0}, 1\right)$.

Iff $\in \mathcal{A}$ satisfies (2.8), then

$$
\mathcal{L}_{c}^{\lambda+1} f(z) \prec q(z)
$$

Proof The proof is similar to that of [1, Theorem 2.3d] and so is omitted.

The next theorem yields the best dominant of the differential subordination (2.7).

Theorem 2.5 Let $h$ be univalent in $\mathbb{U}$ and let $\phi: \mathbb{C}^{3} \times \mathbb{U} \times \overline{\mathbb{U}} \rightarrow \mathbb{C}$. Suppose that the differential equation

$$
\phi\left(q(z), \frac{z q^{\prime}(z)+(c-1) q(z)}{c}, \frac{z^{2} q^{\prime \prime}(z)+(2 c-1) z q^{\prime}(z)+(c-1)^{2} q(z)}{c^{2}} ; z, \xi\right)=h(z)
$$

has a solution $q$ with $q(0)=0$ and satisfies one of the following conditions:

(1) $q \in \mathcal{Q}_{0}$ and $\phi \in \Phi_{\mathcal{L}}[h, q]$,

(2) $q$ is univalent in $\mathbb{U}$ and $\phi \in \Phi_{\mathcal{L}}\left[h, q_{\rho}\right]$ for some $\rho \in(0,1)$, or

(3) $q$ is univalent in $\mathbb{U}$ and there exists $\rho_{0} \in(0,1)$ such that $\phi \in \Phi_{\mathcal{L}}\left[h_{\rho}, q_{\rho}\right]$ for all $\rho \in\left(\rho_{0}, 1\right)$.

Iff $\in \mathcal{A}$ satisfies (2.8) and

$$
\phi\left(\mathcal{L}_{c}^{\lambda+1} f(z), \mathcal{L}_{c}^{\lambda} f(z), \mathcal{L}_{c}^{\lambda-1} f(z) ; z, \xi\right)
$$

is analytic in $\mathbb{U}$, then

$$
\mathcal{L}_{c}^{\lambda+1} f(z) \prec q(z)
$$

and $q$ is the best dominant.

Proof Following the same arguments as in [1, Theorem 2.3e], we deduce that $q$ is a dominant from Theorem 2.2 and Theorem 2.4. Since $q$ satisfies (2.9), it is also a solution of (2.8) and therefore $q$ will be dominated by all dominants. Hence, $q$ is the best dominant. 
In the particular case $q(z)=M z, M>0$, and in view of Definition 2.1, the class of admissible functions $\Phi_{\mathcal{L}}[\Omega, q]$, denoted by $\Phi_{\mathcal{L}}[\Omega, M]$, is described below.

Definition 2.2 Let $\Omega$ be a set in $\mathbb{C}, \operatorname{Re}\{c\}>0, \lambda \geq 1$ and $M>0$. The class of admissible functions $\Phi_{\mathcal{L}}[\Omega, M]$ consists of those functions $\phi: \mathbb{C}^{3} \times \mathbb{U} \times \overline{\mathbb{U}} \rightarrow \mathbb{C}$ such that

$$
\phi\left(M e^{i \theta}, \frac{(k+c-1) M e^{i \theta}}{c}, \frac{L+\left[(2 c-1) k+(c-1)^{2}\right] M e^{i \theta}}{c^{2}} ; z, \xi\right) \notin \Omega,
$$

whenever $z \in \mathbb{U}, \xi \in \overline{\mathbb{U}}, \operatorname{Re}\left\{L e^{i \theta}\right\} \geq(k-1) k M, \theta \in \mathbb{R}$ and $k \geq 1$.

Corollary 2.6 Let $\phi \in \Phi_{\mathcal{L}}[\Omega, M]$. If $\in \mathcal{A}$ satisfies

$$
\phi\left(\mathcal{L}_{c}^{\lambda+1} f(z), \mathcal{L}_{c}^{\lambda} f(z), \mathcal{L}_{c}^{\lambda-1} f(z) ; z, \xi\right) \in \Omega
$$

then

$$
\mathcal{L}_{c}^{\lambda+1} f(z) \prec M z
$$

In the special case $\Omega=q(\mathbb{U})=\{w:|w|<M\}$, the class $\Phi_{\mathcal{L}}[\Omega, M]$ is simply denoted by $\Phi_{\mathcal{L}}[M]$.

Corollary 2.7 Let $\phi \in \Phi_{\mathcal{L}}[M]$. If $f \in \mathcal{A}$ satisfies

$$
\left|\phi\left(\mathcal{L}_{c}^{\lambda+1} f(z), \mathcal{L}_{c}^{\lambda} f(z), \mathcal{L}_{c}^{\lambda-1} f(z) ; z, \xi\right)\right|<M
$$

then

$$
\left|\mathcal{L}_{c}^{\lambda+1} f(z)\right|<M
$$

Corollary 2.8 Let $c>1, M>0$ and let $C(\xi)$ be an analytic function in $\overline{\mathbb{U}}$ with $\operatorname{Re}\{\zeta C(\xi)\} \geq$ 0 for $\zeta \in \partial \mathbb{U}$. Iff $\in \mathcal{A}$ satisfies

$$
\left|c^{2} \mathcal{L}_{c}^{\lambda-1} f(z)-c \mathcal{L}_{c}^{\lambda} f(z)-(c-1)^{2} \mathcal{L}_{c}^{\lambda+1} f(z)+C(\xi)\right|<(c-1) M
$$

then

$$
\left|\mathcal{L}_{c}^{\lambda+1} f(z)\right|<M
$$

Proof This follows from Corollary 2.6 by taking $\phi(u, v, w ; z, \xi)=c^{2} w-c v-(c-1)^{2} u+C(\xi)$ and $\Omega=h(\mathbb{U})$, where $h(z)=(c-1) M z$. To use Corollary 2.6, we need to show that $\phi \in$ $\Phi_{\mathcal{L}}[\Omega, M]$, that is, the admissible condition (2.10) is satisfied. This follows since

$$
\begin{aligned}
& \left|\phi\left(M e^{i \theta}, \frac{(k+c-1) M e^{i \theta}}{c}, \frac{L+\left[(2 c-1) k+(c-1)^{2}\right] M e^{i \theta}}{c^{2}} ; z, \xi\right)\right| \\
& \quad=\left|L+\left[(2 c-1) k+(c-1)^{2}\right] M e^{i \theta}-(k+c-1) M e^{i \theta}-(c-1)^{2} M e^{i \theta}+C(\xi)\right| \\
& \quad=\left|L+(2 k-1)(c-1) M e^{i \theta}+C(\xi)\right|
\end{aligned}
$$




$$
\begin{aligned}
& \geq(2 k-1)(c-1) k M+\operatorname{Re}\left\{L e^{-i \theta}+\operatorname{Re}\left\{C(\xi) e^{-i \theta}\right\}\right\} \\
& \geq(2 k-1)(c-1) M+k(k-1) M+\operatorname{Re}\left\{C(\xi) e^{-i \theta}\right\} \\
& \geq(c-1) M
\end{aligned}
$$

for $z \in \mathbb{U}, \xi \in \overline{\mathbb{U}}, \operatorname{Re}\left\{L e^{-i \theta}\right\} \geq(k-1) k M, \theta \in \mathbb{R}$ and $k \geq 1$. Hence, by Corollary 2.6, we deduce the required results.

\section{Superordination and sandwich-type results}

The dual problem of differential subordination, that is, differential superordination of the Komatu integral operator $\mathcal{L}_{c}^{\lambda}$ defined by (1.1), is investigated in this section. For this purpose, the class of admissible functions is given in the following definition.

Definition 3.1 Let $\Omega$ be a set in $\mathbb{C}, q \in \mathcal{H}[0,1]$ with $q^{\prime}(z) \neq 0, \operatorname{Re}\{c\}>0$ and $\lambda \geq 1$. The class of admissible functions $\Phi_{\mathcal{L}}^{\prime}[\Omega, q]$ consists of those functions $\phi: \mathbb{C}^{3} \times \overline{\mathbb{U}} \times \overline{\mathbb{U}} \rightarrow \mathbb{C}$ that satisfy the admissibility condition

$$
\phi(u, v, w ; \zeta, \xi) \in \Omega
$$

whenever

$$
u=q(z), \quad v=\frac{z q^{\prime}(z) / m+(c-1) q(z)}{c}
$$

and

$$
\operatorname{Re}\left\{\frac{c^{2} w-(c-1)^{2} u}{c v-(c-1) u}-2(c-1)\right\} \leq \frac{1}{m} \operatorname{Re}\left\{\frac{z q^{\prime \prime}(z)}{q^{\prime}(z)}+1\right\}
$$

for $z \in \mathbb{U}, \zeta \in \partial \mathbb{U}, \xi \in \overline{\mathbb{U}}$ and $m \geq 1$.

Theorem 3.1 Let $\phi \in \Phi_{\mathcal{L}}^{\prime}[\Omega, q]$. If $f \in \mathcal{A}, \mathcal{L}_{c}^{\lambda+1} f(z) \in \mathcal{Q}_{0}$ and

$$
\phi\left(\mathcal{L}_{c}^{\lambda+1} f(z), \mathcal{L}_{c}^{\lambda} f(z), \mathcal{L}_{c}^{\lambda-1} f(z) ; z, \xi\right)
$$

is univalent in $\mathbb{U}$, then

$$
\Omega \subset\left\{\phi\left(\mathcal{L}_{c}^{\lambda+1} f(z), \mathcal{L}_{c}^{\lambda} f(z), \mathcal{L}_{c}^{\lambda} f(z) ; z, \xi\right): z \in \mathbb{U}, \xi \in \overline{\mathbb{U}}\right\}
$$

implies

$$
q(z) \prec \mathcal{L}_{c}^{\lambda+1} f(z)
$$

Proof From (2.7) and (3.1), we have

$$
\Omega \subset\left\{\psi\left(p(z), z p^{\prime}(z), z^{2} p^{\prime \prime}(z) ; z, \xi\right): z \in \mathbb{U}, \xi \in \overline{\mathbb{U}}\right\} .
$$


From (2.5), we see that the admissibility condition for $\phi \in \Phi_{\mathcal{L}}^{\prime}[\Omega, q]$ is equivalent to the admissibility condition for $\psi$ as given in Definition 1.2. Hence, $\psi \in \Psi^{\prime}[\Omega, q]$, and by Theorem $1.2, q \prec p$ or

$$
q(z) \prec \mathcal{L}_{c}^{\lambda+1} f(z),
$$

which evidently completes the proof of Theorem 3.1.

If $\Omega \neq \mathbb{C}$ is a simply connected domain, then $\Omega=h(\mathbb{U})$ for some conformal mapping $h$ of $\mathbb{U}$ onto $\Omega$. In this case, the class $\Phi_{\mathcal{L}}^{\prime}[h(\mathbb{U}), q]$ is written as $\Phi_{\mathcal{L}}^{\prime}[h, q]$. Proceeding similarly as in the previous section, the following result is an immediate consequence of Theorem 3.1.

Theorem 3.2 Let $q \in \mathcal{H}[0,1], h$ be analytic in $\mathbb{U}$ and $\phi \in \Phi_{\mathcal{L}}^{\prime}[h, q]$. If $f(z) \in \mathcal{A}, \mathcal{L}_{c}^{\lambda+1} f(z) \in$ $\mathcal{Q}_{0}$ and

$$
\phi\left(\mathcal{L}_{c}^{\lambda+1} f(z), \mathcal{L}_{c}^{\lambda} f(z), \mathcal{L}_{c}^{\lambda-1} f(z) ; z, \xi\right)
$$

is univalent in $\mathbb{U}$, then

$$
h(z) \prec \prec \phi\left(\mathcal{L}_{c}^{\lambda+1} f(z), \mathcal{L}_{c}^{\lambda} f(z), \mathcal{L}_{c}^{\lambda-1} f(z) ; z, \xi\right)
$$

implies

$$
q(z) \prec \mathcal{L}_{c}^{\lambda+1} f(z)
$$

Theorem 3.1 and Theorem 3.2 can only be used to obtain subordinants of differential superordination of the form (3.1) or (3.2). The following theorem proves the existence of the best subordinant of (3.2) for certain $\phi$.

Theorem 3.3 Let $h$ be analytic in $\mathbb{U}$ and $\phi: \mathbb{C}^{3} \times \mathbb{U} \times \overline{\mathbb{U}} \rightarrow \mathbb{C}$. Suppose that the differential equation

$$
\phi\left(q(z), \frac{z q^{\prime}(z)+(c-1) q(z)}{c}, \frac{z^{2} q^{\prime \prime}(z)+(2 c-1) z q^{\prime}(z)+(c-1)^{2} q(z)}{c^{2}} ; z, \xi\right)=h(z)
$$

has a solution $q \in \mathcal{Q}_{0}$. If $\phi \in \Phi^{\prime}{ }_{\mathcal{L}}[h, q], f \in \mathcal{A}, \mathcal{L}_{c}^{\lambda+1} f(z) \in \mathcal{Q}_{0}$ and

$$
\phi\left(\mathcal{L}_{c}^{\lambda+1} f(z), \mathcal{L}_{c}^{\lambda} f(z), \mathcal{L}_{c}^{\lambda-1} f(z) ; z, \xi\right)
$$

is univalent in $\mathbb{U}$, then

$$
h(z) \prec \prec \phi\left(\mathcal{L}_{c}^{\lambda+1} f(z), \mathcal{L}_{c}^{\lambda} f(z), \mathcal{L}_{c}^{\lambda-1} f(z) ; z, \xi\right)
$$

implies

$$
q(z) \prec \mathcal{L}_{c}^{\lambda+1} f(z),
$$

and $q$ is the best subordinant. 
Proof The proof is similar to that of Theorem 2.5 and so is omitted.

Combining Theorem 2.2 and Theorem 3.2, we obtain the following sandwich-type theorem.

Theorem 3.4 Let $h_{1}$ and $q_{1}$ be analytic functions in $\mathbb{U}, h_{2}$ be a univalent function in $\mathbb{U}, q_{2} \in$ $\mathcal{Q}_{0}$ with $q_{1}(0)=q_{2}(0)=0$ and $\phi \in \Phi_{\mathcal{L}}\left[h_{2}, q_{2}\right] \cap \Phi_{\mathcal{L}}^{\prime}\left[h_{1}, q_{1}\right]$. Iff $\in \mathcal{A}, \mathcal{L}_{c}^{\lambda+1} f(z) \in \mathcal{H}[0,1] \cap \mathcal{Q}_{0}$ and

$$
\phi\left(\mathcal{L}_{c}^{\lambda+1} f(z), \mathcal{L}_{c}^{\lambda} f(z), \mathcal{L}_{c}^{\lambda-1} f(z) ; z, \xi\right)
$$

is univalent in $\mathbb{U}$, then

$$
h_{1}(z) \prec \prec \phi\left(\mathcal{L}_{c}^{\lambda+1} f(z), \mathcal{L}_{c}^{\lambda} f(z), \mathcal{L}_{c}^{\lambda-1} f(z) ; z, \xi\right) \prec \prec h_{2}(z)
$$

implies

$$
q_{1}(z) \prec \mathcal{L}_{c}^{\lambda+1} f(z) \prec q_{2}(z) .
$$

\section{Competing interests}

The author declares that they have no competing interests.

\section{Authors' contributions}

The author worked on the results and he read and approved the final manuscript.

\section{Acknowledgements}

Dedicated to Professor Hari M Srivastava.

This research was supported by the Basic Science Research Program through the National Research Foundation of Korea (NRF) funded by the Ministry of Education, Science and Technology (No. 2012-0002619).

\section{Received: 27 November 2012 Accepted: 21 January 2013 Published: 4 March 2013}

\section{References}

1. Miller, SS, Mocanu, PT: Differential Subordination, Theory and Application. Marcel Dekker, New York (2000)

2. Komatu, Y: Distortion Theorems in Relation to Linear Integral Operators. Kluwer Academic, Dordrecht (1996)

3. Flett, TM: The dual of an inequality of Hardy and Littlewood and some related inequalities. J. Math. Anal. Appl. 38, 746-765 (1972)

4. Jung, IB, Kim, YC, Srivastava, HM: The Hardy space of analytic functions associated with certain one-parameter families of integral operators. J. Math. Anal. Appl. 176, 138-147 (1993)

5. Liu, JL: A linear operator and strongly starlike functions. J. Math. Soc. Jpn. 54, 975-981 (2002)

6. Antonino, JA: Strong differential subordination to Briot-Bouquet differential equations. J. Differ. Equ., 114: 101-105 (1994)

7. Antonino, JA: Strong differential subordination and applications to univalency conditions. J. Korean Math. Soc. 43 , 311-322 (2006)

8. Oros, Gl, Oros, G: Strong differential subordination. Turk. J. Math. 33, 249-257 (2009)

9. Oros, Gl: Strong differential superordination. Acta Univ. Apulensis, Mat.-Inform. 19, 101-106 (2009)

10. Miller, SS, Mocanu, PT: Subordinants of differential superordinations. Complex Var. Theory Appl. 48, $815-826$ (2003)

11. Ali, RM, Ravichandran, V, Seenivasagan, N: Subordination and superordination of the Liu-Srivastava operator on meromorphic functions. Bull. Malays. Math. Soc. 31, 193-207 (2008)

12. Ali, RM, Ravichandran, V, Seenivasagan, N: Differential subordination and superordination of analytic functions defined by the multiplier transformation. Math. Inequal. Appl. 12, 123-139 (2009)

13. Ali, RM, Ravichandran, V, Seenivasagan, N: Differential subordination and superordination of analytic functions defined by the Dziok-Srivastava linear operator. J. Franklin Inst. 347, 1762-1781 (2010)

14. Ali, RM, Ravichandran, V, Seenivasagan, N: On subordination and superordination of the multiplier transformation of meromorphic functions. Bull. Malays. Math. Soc. 33, 311-324 (2010)

15. Cho, NE, Kwon, OS, Srivastava, HM: Strong differential subordination and superordination for multivalently meromorphic functions involving the Liu-Srivastava operator. Integral Transforms Spec. Funct. 21, 589-601 (2010) 